José Manuel Macário Rebêlo 1

Vera Lúcia Lopes de Barros 1

Walter Araújo Mendes 2

\title{
Espécies de Triatominae (Hemiptera: Reduviidae) do Estado do Maranhão, Brasil
}

\author{
Triatominae species (Hemiptera: Reduviidae) \\ in Maranhão State, Brazil
}

\footnotetext{
1 Departamento de Patologia, Núcleo de Patologia Tropical e Medicina Social, Universidade Federal do Maranhão. Praça Madre Deus 2, São Luís, MA 65025-560, Brasil.

2 Fundação Nacional de Saúde. Rua 5 de Janeiro s/no, São Luís, MA 65040-450, Brasil.
}

\begin{abstract}
This paper provides a checklist of 15 known species of the Triatominae subfamily in Maranhão State. On the coastal São Luís Island there are a total of 10 species associated with sylvatic areas (Eratyrus mucronatus, Psammolestes tertius, Panstrongylus diasi) and periurban and urban areas (Panstrongylus geniculatus, Panstrongylus lignarius, Rhodnius nasutus, Rhodnius neglectus, Rhodnius pictipes, Rhodnius robustus and Triatoma rubrofasciata). The last was found only on São Luís Island, whilethe others have also been found inland. The following were not found in the coastal area: Panstrongylus megistus, Triatoma brasiliensis, Triatoma maculata, Triatoma pseudomaculata and Rhodnius brethesi. These were found mainly on the "Chapadas" and "Chapadões" areas in the eastern and southern parts of the State.

Key words Triatominae; Chagas Disease; Insect Vectors
\end{abstract}

Resumo Cerca de 15 espécies de Triatominae são de conhecida ocorrência no Estado do Maranhão. Na Il ha de São Luís, zona do litoral, dez espéci es já foram encontradas associadas às áreas silvestres: Eratyrus mucronatus, Psammolestes tertius, Panstrongylus diasi, e às habitações das áreas urbanas e periurbanas: Panstrongylus geniculatus, Panstrongylus lignarius, Rhodnius nasutus, Rhodnius neglectus, Rhodnius pictipes, Rhodnius robustus e Triatoma rubrofasciata. Esta última tem sido encontrada exclusi vamente na Il ha de São Luís, ao passo que as demais também ocorrem no interior do Estado. Na zona litorânea, não foram encontradas: Panstrongylus megistus, Triatoma brasiliensis, Triatoma maculata, Triatoma pseudomaculata e Rhodnius brethesi, as quais são encontradas nas áreas mais secas das zonas que formam as chapadas e os chapadões do Sudeste e Sul do Estado.

Palavras-chave Triatominae; Doença de Chagas; Insetos Vetores 


\section{Introdução}

Com exceção do gênero Linshcosteus e algumas espécies do gênero Triatoma, os triatomíneos são exclusivos do Novo Mundo, distribuindo-se desde os Estados Unidos até a Argentina, sendo mais diversos na região neotropical (Lent \&Wygodzinsky, 1979; Alencar, 1980). A subfamília subdivide-se em cinco tribos, 14 gêneros e cerca de 115 espécies descritas (Jurberg \& Galvão, 1997).

A importância epidemiológica do grupo reside na transmissão do Trypanosoma cruzi ao homem. Embora todas as espécies de triatomíneos sejam vetores em potencial deste protozoário, apenas aquel as que colonizam no domicílio e ou peridomicílio reúnem condições necessárias para transmitir a doença de Chagas humana. Neste aspecto, os gêneros de maior importância epidemiológica são: Panstrongylus, Triatoma e Rhodnius.

No Brasil, das 41 espécies de triatomíneos conhecidas, apenas nove destacam-se como vetores da doença de Chagas: T. infestans, P. megistus, T. brasiliensis T. pseudomaculata, T. vitticeps, T. sordida, T. rubrofasciata, R. neglectus (Corrêa, 1968) e R. prolixus, encontrada naturalmente infectada no Amazonas, Pará, Minas Gerais e São Paulo (Ferraz et al., 1975).

Apesar da grande importância dos triatomíneos como vetores da doença de Chagas, doença endêmica em várias regiões do Brasil, incluindo o Nordeste, pouco se sabe a respeito da fauna do Maranhão, tendo-se conhecimento apenas do trabalho de Brazil et al. (1985), o qual apresenta dados sobre a distribuição e infecção dos triatomíneos da Ilha de São Luís.

Neste estudo, demonstram-se al guns dados preliminares sobre a diversidade e padrão de distribuição dos triatomíneos no Estado do Maranhão.

\section{Metodologia}

O presente estudo consistiu de um levantamento das espécies de Triatominae que se distribuem no Estado do Maranhão, tendo por base dados fornecidos pela Fundação Nacional de Saúde (FNS-MA), sendo complementado com informações relatadas na literatura.

A seguir faz-se um breve comentário sobre os aspectos físico-climatológicos do Maranhão, para melhor compreensão do padrão de distribuição desses reduvídeos no Estado.

O Estado do Maranhão, com uma superfície de $328.663 \mathrm{~km}^{2}$ ou $3,86 \%$ do território nacional, localiza-se no Nordeste Ocidental do
Brasil, entre $01^{\circ} 01^{\prime}$ e $10^{\circ} 21^{\prime} 07^{\prime \prime}$ LS e $41^{\circ} 48^{\prime}$ $30^{\prime \prime}$ e $48^{\circ} 50^{\prime}$ 51" LW. Na sua parte setentrional possui $640 \mathrm{~km}$ de costa. Cerca de $1.365 \mathrm{~km}$ do seu território faz fronteira, a Leste, com o Estado do Piauí, separando-se dele pelo Rio Parnaíba; a Noroeste, são $798 \mathrm{~km}$ de fronteira com o Estado do Pará, o Rio Gurupi marcando a região limítrofe entre os dois Estados. A Sudoeste, a linha fronteiriça com o Estado de Tocantins é de 1.060 km e o Rio Manoel Alves Grande constitui a linha divisória (IBGE, 1984).

O clima predominante é o quente e úmido, apresentando durante todo o ano temperaturas elevadas, em média $27^{\circ} \mathrm{C}$, com pequenas variações. Na zona que inclui as regiões ecológicas da Pré-Amazônia e da Baixada Ocidental, a Oeste do Rio Mearim, é onde domina o clima tropical superúmido ou equatorial, com índice pluviométrico elevado, em torno de $2.000 \mathrm{~mm}$ anuais de chuvas, e temperatura média anual de $25^{\circ} \mathrm{C}$, com estação seca pouco nítida. No resto do Estado, distinguem-se duas estações bem definidas: uma seca, de julho a dezembro, e outra chuvosa, de janeiro a junho. No Leste e Sudeste do Estado, nas Zonas dos Cerrados e Chapadões, os índices pluviométricos são mais baixos, chegando a $1.200 \mathrm{~mm}$ anuais de chuvas (IBGE, 1984).

O relevo é moderado, com altitudes médias variando entre $200 \mathrm{~m}$ e $400 \mathrm{~m}$, tendo o ponto mais alto - o Monte Mearim - entre as Serras da Canela e Negra, na cabeceira do Rio Mearim, com cerca de $1.300 \mathrm{~m}$ de altitude.

As bacias hidrográficas mais importantes são as do: 1) Gurupi, com cerca de 40.000 km² de superfície, tendo o Gurupi como principal rio, com 800 km de extensão; 2) Turiaçu, com $32 \mathrm{~km}^{2}$ de superfície, a Noroeste do Estado, tendo o rio de mesmo nome cerca de $700 \mathrm{~km}$ de extensão; 3) Golfão Maranhense, compreendendo os seguintes rios: Mearim, Grajaú, Pindaré, Munim eltapecuru; 4) Parnaíba, que faz, em toda sua extensão, fronteira com o Piauí e o Maranhão, possuindo dois afluentes pelo lado maranhense - rios das Balsas (720 km) e da Babilônia (311km) (IBGE, 1984).

O Estado do Maranhão é rico em ecossistemas florísticos, podendo-se destacar as seguintes zonas ecológicas (Figura 1):

\section{Zona do Litoral}

Localiza-se na parte mais setentrional do Estado destacando-se pela presença de mangues (encontrados desde a fronteira com o Pará até a Baía de São Marcos), restingas, coqueirais e campos, que aparecem nas partes baixas em torno do Golfão Maranhense. As dunas predo- 
minam no litoral oriental do Estado e são a principal atração do Parque Nacional dos Lençóis Maranhenses.

\section{Pré-Amazônia}

Ocupa a parte centro-ocidental do Estado, incluindo as áreas do Médio Grajaú e parte do Vale do Rio Pindaré; é o domínio das florestas, originalmente representadas pelas castanheira (Bertholletia excelsa H.B.K.), seringueira (Hevea brasiliensis Muell. Arg.), piquiá (Aspidosperma sessiliflorum Mart.), camuru, mumuru, peroba (Aspidosperma polyneuron Mull. Arg.), jacarandá (Machaerium villosum Vog.). Nas áreas de concavidade, as representantes são as palmeiras de juçara (Euterpe edulis Mart.) e buriti (Mauritia vinifera Mart.).

\section{Zona dos Cocais}

Ocupa o centro-oriental, notadamente os vales dos rios Itapecuru e Mearim. É o domínio das Matas dos Cocais, destacando-se o babaçual (Orbignya martiana B.R.), secundado por outras palmeiras, como tucum (Bactris setosa Mart.) e marajaíba.

Nas duas últimas zonas, as formações vegetais desenvolvem-se sobre sedimentos da Formação Itapecuru do Cretáceo Inferior, constituído de arenitos finos, recobrindo as formações mais antigas.

Na região de contato entre a Zona dos Cocais e dos Cerrados, no Vale do Parnaíba, predominam as florestas mistas - Floresta Estacional Perenifólia Aberta com Babaçu, Carnaúba (Copernicia prunifera (Mill.) Moore) e Manchas de Cerrados.

Os cerrados ocupam grande parte do território maranhense, recobrindo toda a região oriental e meridional do Estado, formando duas zonas distintas: os Chapadões e os Cerrados.

\section{Zona dos Cerrados e dos Chapadões}

Estão presentes nas nascentes dos rios Grajaú e Mearim e nos altos cursos dos rios das Balsas e Parnaíba. Nessa vegetação, destacam-se capotão, pequizeiro (Caryocar brasiliensis Camb.), jatobá (Hymenaea courbaril Lin.), andiroba (Carapa guianensis Aubl.) etc. Os sedimentos dessas áreas pertencem às formações Corda, Sambaíba, Pedra de Fogo e Piauí, de origem mais antiga (Carbonífero-Jurássico), e al guns trechos das formações Pastos Bons e Sardinha.

Manchas de caatingas podem ser observadas no Baixo Parnaiba, formando zonas de contato com a Floresta Perenifólia Aberta da Bai-
Figura 1

Distribuição dos Triatominae no Estado do Maranhão, de acordo com a zona ecológica.

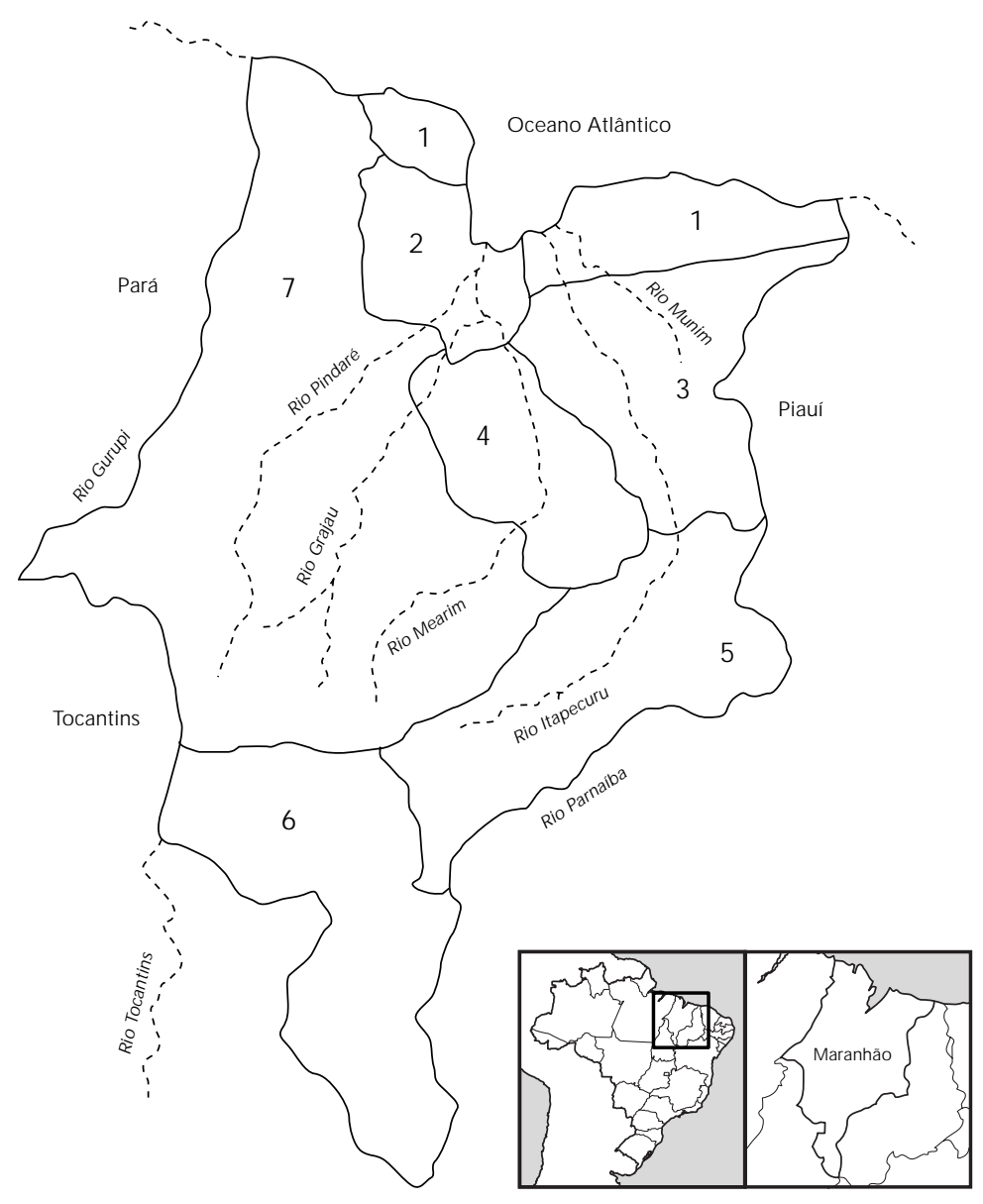

1 - Litoral: E. mucronatus, P. diasi, P. lignarius, $P$. geniculatus, $P$. tertius, $R$. nasutus,

$R$. neglectus, $R$. pictipes, $R$. robustus, T. rubrofasciata.

2 - Baixada: E. mucronatus, P. lignarius, P. tertius.

3 - Cerrado: P. diasi, P. megistus, $R$. neglectus, $R$. pictipes, $R$. robustus,

T. pseudomaculata.

4 - Cocais: $R$. nasutus, $R$. neglectus, $R$. pictipes.

5 - Chapadões: P. megistus, R. brethesi, R. nasutus, R. neglectus, R. pictipes,

T. brasiliensis, T. maculata, T. pseudomaculata.

6 - Planalto: P. geniculatus, P. megistus, R. neglectus, R. pictipes, T. brasiliensis,

T. pseudomaculata.

7 - Pré-Amazônia: $R$. neglectus, R. pictipes, T. pseudomaculata.

xada Oriental e com o Cerrado nas proximidades do Piauí; não apresenta abundância de cactácea, que dá o aspecto rude à vegetação nordestina, sendo dominado pelo sabiá, o paud'arco (Tecoma heptaphylla Mart.), a catingade-porco, a aroeira e o jatobá, entre outros.

\section{Zona do Planalto}

Constitui o prolongamento dos chapadões do Estado de Goiás. 


\section{Resultados e discussão}

São 15 as espécies de Triatominae assinaladas no Estado do Maranhão: Eratyrus mucronatus (Stal, 1859); Psammolestestertius (Lent \& Jurberg, 1965); Panstrongylus diasi (Pinto \& Lent, 1946); Panstrongylus geniculatus (Latreille, 1811); Panstrongylus lignarius (Walker, 1837); Panstrongylus megi stus (Burmeister, 1835); Rhodnius brethesi (Matta, 1919); Rhodnius nasutus (Stal, 1859); Rhodnius neglectus (Lent, 1954); Rhodnius pictipes (Stal, 1872); Rhodnius robustus(Larrousse, 1927); Triatoma brasiliensis (Neiva, 1911); Triatoma maculata (Erickson, 1848); Triatoma pseudomaculata (Correa \& Espinola, 1964); Triatoma rubrofasciata (De Geer, 1773).

Entre as espécies incriminadas como vetores da doença de Chagas no Brasil, cinco são encontradas no Maranhão: P. megistus, T. brasiliensis, T. pseudomaculata, T. rubrofasciata e R. neglectus. Estas espécies apresentam padrão de distribuição diverso no extenso território do Estado.

Na Ilha de São Luís, algumas espécies estão intimamente associadas aos ambientes silvestres (E. mucronatus, P. diasi e P. tertius), outras são encontradas associadas com as habitações periurbanas (P. geniculatus, R. neglectus, R. robustus) e urbanas (P. lignarius, R. nasutus, $R$. pictipes, T. rubrofasciata). Não ocorrem nesta zona, P. megistus, T. brasiliensis, T. maculata, T. pseudomaculata e R. brethesi, encontradas apenas no interior do Estado.

E. mucronatus distribui-se nos Estados do Amazonas, Pará e Mato Grosso. No Maranhão, é encontrada na Zona da Baixada Ocidental, a exemplo de P. tertius. Esta última espécie tem registro no Estado do Mato Grosso e, no Maranhão, associa-se com ninhos de xexéu - Cacicus cela (Lin.). Associa-se também com ninhos de aves da família Furnariidae e Psittacidae, não tendo importância na epidemiologia da doença de Chagas (Alencar, 1980).

P. megistus tem ampla distribuição no Brasil, ocorrendo desde o Rio Grande do Sul até o Pará (Alencar, 1980; Schofield, 1985). É, juntamente com $\mathrm{T}$. infestans, a espécie mais importante na transmissão de Chagas no Brasil. Existem registros de sua ocorrência também na Argentina, Paraguai e Bolívia. No Maranhão, tem sido encontrada, principalmente, nas áreas secas da Zona dos Chapadões do Baixo Balsas e do Alto Itapecuru. Esta espécie pode circular em ambiente peridomiciliar e se aproximar do homem, eventualmente invadindo as habitações destes (Garcia-Zapata \& Marsden, 1986) e formando colônias permanentes (Barreto, 1979;). No Estado do Pará, coloniza, para sugar, em to- dos os ecótopos peridomiciliares, onde encontra o cão, gato, roedores, cabra, galinhas, e no intradomicílio, mais intensamente no quarto e nos depósitos, onde encontra o homem (Alencar, 1987). Nas áreas silvestres, pode ser encontrada em copas de palmeira macaubeira (Lent $\&$ Wygodzinsky, 1979). No seu ambiente natural, tem desenvolvido hábito marsupiófilo e dendrífilo (Alencar, 1987). Em Goiás, poucas ninfas tem sido encontradas dentro de casa (Garcia-Zapata \& Marsden, 1986).

P. geniculatus foi encontrada em São Luís e Imperatriz, na Amazônia Maranhense. É uma espécie essencialmente silvestre, vivendo comumente em tocas de tatus, em toda a região neotropical. Existe registro de ocorrência desta espécie também na copa de palmeira (Lent \& Wygodzinsky, 1979), podendo ali desenvolver hábito quiroptófilo. Os adultos podem invandir ecótopos artificiais.

P. lignarius é comum no Norte do Estado, incluindo a Baixada Ocidental, e, na llha de São Luís, é a espécie de distribuição mais homogênea.

P. diasi distribui-se desde o Estado da Bahia até São Paulo, e também em Minas Gerais e Goiás. No Maranhão, os poucos registros assinalam sua presença na Ilha de São Luís e Zona dos Cerrados do Alto Munim.

R. negl ectus é uma espécie típica das savanas do Brasil central, distribuindo-se nos Estados da Bahia, Minas Gerais, São Paulo, Goiás e Mato Grosso. É ornitófila, encontrada em palmeiras de macaubeira, ouricuri, buriti, babaçu associada aos ninhos de aves, Annumbius annumbi. Infecta-se e é capaz de colonizar as habitações humanas (Lent \& Wygodzinsky, 1979). No Maranhão, tem sido encontrada desde a Zona do Planalto, ao Sul, até a Il ha de São Luís, passando pelas Zonas dos Chapadões, Cerrados e Cocais, não sendo encontrada nas áreas de influência amazônica, como Pré-Amazônia maranhense e Baixada Ocidental.

R. robustus, R. pictipes e R. nasutus restringem-se ao Norte do Brasil (Lent \& Wygodzinsky, 1979; Alencar, 1980).

R. robustus distribui-se nos Estados do Amazonas e Pará. É ornitófila e se infecta (Alencar, 1980). No Maranhão, tem sido encontrada na Zona dos Cerrados do Nordeste do Estado e na Ilha de São Luís.

R. pictipes também ocorre no Amazonas e Pará; no Maranhão, é a espécie de distribuição mais uniforme, ocorrendo em todas as zonas ecológicas, penetrando, inclusive, no vizinho Estado do Piauí. É arborícola, associa-se com ninhos de morcegos em palmeiras e se infecta (Alencar, 1980). 
R. nasutus estende sua distribuição numa faixa mais ampla, desde o Estado do Pará até o Rio Grande do Norte, passando pelo Piauí e Ceará; no Maranhão, ocorre na Ilha de São Luís, Zona do Litoral, associada com ninhos de pombos no intra e peridomicílios; pode ser encontrada nos ecótopos artificiais. Ao contrário de seus congêneres, que penetram nas áreas mais secas do Sul do Estado, esta espécie tem sido encontrada com mais freqüência, na porção centro-norte, incluindo as áreas amazônicas e os Chapadões do Alto Itapecuru, Zona dos Cocais e Il ha de São Luís. No Ceará, infecta-se em taxas muito baixas (1,3\%) (Alencar, 1987); associa-se com aves em abrigos semidomiciliares e ninhos silvestres, com marsupiais, roedores e morcegos.

T. pseudomaculata ocorre em todos os estados nordestinos, não tendo registro apenas para Sergipe. Foi assinalada também em Minas Gerais e Goiás. É encontrada em ecótopos naturais e artificiais e, mesmo não sendo uma espécie transmissora habitual, pode, ocasionalmente, adentrar nos domicílios e vir a adquirir esta qualidade. É ornitófila, associando-se freqüentemente com ninhos de Nimbus saturnus e com galinheiros (Alencar, 1980). No domicílio, pode ser encontrada nos diversos cômodos e telhados. No Maranhão, tem ocorrência conhecida no interior, em todas as zonas ecológicas, não sendo encontrada apenas na llha de São Luís e demais áreas litorâneas.

T. maculata tem sido encontrada em Roraima, Goiás e Maranhão. É essencialmente silvestre e ornitófila, mas pode ser encontrada no peridomicílio (Alencar, 1980). No Maranhão, tem-se registro de sua ocorrência nos Chapadões do Alto Itapecuru.

R. brethesi foi assinalada nas áreas úmidas do Brasil: no Amazonas e Pará, associada com roedores. No Maranhão, tem sido encontrada em áreas mais secas, notadamente nas Zonas dos Chapadões do Baixo Balsas e do Alto Itapecuru.

T. brasiliensis é o principal vetor de Chagas no polígono da seca. Tem ampla distribuição no nordeste brasileiro, ocorrendo em todos os Estados, não tendo registro apenas para Sergipe (Schofield, 1985). É encontrada também em Minas Gerais e Goiás. No Maranhão, é encontrada na Zona dos Chapadões, no Município de São Francisco do Maranhão, nas proximidades do M édio Parnaíba. Ocorre em ecótopos naturais e artificiais, apresentando os mais diversos hábitos: quiropterófilo, dendrífilo, ornitófilo e rodentófilo. Pode atacar o homem e animais durante o dia, quando faminta. No domicílo, a preferência é maior por galinha, gato, cabra, cão, sugando menos o rato, o porco, o marsupial e o boi (Alencar, 1980).

T. rubrofasciata pode ser encontrada em toda a região costeira dos trópicos, intimamente associada com o roedor Rattus rattus rattus, transmitindo-Ihe o Trypanosoma conorrhini (Espínola \& Diotaiuti, 1991). Ambos provavelmente foram introduzidos no continente americano oriundos da Índia. Embora seja uma espécie altamente domiciliar, é considerada como vetor secundário de Chagas no Brasil. Na Ilha de São Luís, tem sido encontrada nas áreas urbanas, inclusive formando colônias dentro dos domicílios, especialmente nos bairros periféricos da capital do Estado, São Luís.

A fauna de triatomíneos no Estado do Maranhão tem sido muito pouco estudada de fato e os dados disponíveis nos mostram a existência das espécies listadas neste trabalho e para as quais procuramos fazer uma breve descrição de sua distribuição no Estado. Entretanto, segundo Brazil et al. (1985) e Marsden et al. (1994), podem ser encontradas ainda no Maranhão as espécies R. prolixus, T. infestans e T. sordida, el evando para 18 o número de espécies presentes no Estado.

Do que foi exposto, podemos concluir que as espécies do gênero Rhodnius apresentaram maior e mais uniforme distribuição no Estado do Maranhão. Aquelas pertencentes ao gênero Panstrongylus mostraram maior afinidade com as áreas da zona litorânea, com exceção de P. megistus, que parece preferir as áreas mais secas dos cerrados e cerradão do centro-sul do Estado. Padrão similar ao desta última espécie foi encontrado para aquelas do gênero Triatoma, com exceção de T. rubrofasciata, cuja distribuição restringe-se às regiões costeiras de todo o trópico, sendo neste estudo encontrada apenas na Il ha de São Luís. 


\section{Agradecimento}

Os autores agradecem à Fundação Nacional de Saúde (MA) pelo fornecimento dos dados aqui apresentados.

\section{Referências}

ALENCAR, J. E., 1980. A Doença de Chagas no Estado do Ceará. Contribui ção para o Conhecimento da sua Epidemiologia Regional. Tese para Professor Titular, Fortaleza: Faculdade de Medicina, Universidade Federal do Ceará.

ALENCAR, J. E., 1987. História Natural da Doença de Chagas no Estado do Ceará. Fortaleza: Imprensa Universitária da UFC.

BRAZIL, R. P.; SILVA, A. R.; ALBARELLI, A. \&VALE, J. F., 1985. Distribuição e infecção de triatomíneos por Trypanosoma do tipo cruzi na Ilha de São Luís-Maranhão. Revista da Sociedade Brasileira de Medicina Tropical, 18:257-260.

BARRETO, M. P., 1979. Ecological aspects of wild reservoirs and vectors of Trypanosoma cruzi. In: Congresso Internacional sobre Doença de Chagas. Rio de Janeiro. (mimeo.).

CORRÊA, R. R., 1968. Informe sobre a doença de Chagas no Brasil e em especial no Estado de São Paulo. Revista Brasileira de Malariologia e Doenças Tropicais, 20:39-81.

ESPÍNOLA, H. N. \& DIOTAIUTI, L., 1991. Hemiptera. In: Parasitologia Humana (D. P Neves \& P. C. Rzezinski, orgs.), 8a ed., pp. 332-349. Rio de Janeiro: Livraria Atheneu Editora.

FERRAZ, D. M.; FERREIRA, E.; RICCIARDI, I. \& NASCIMENTO, C. G., 1975. Chave ilustrada dos triatomíneos transmissores da doença de Chagas, no Brasil. Revista Brasileira de Malariologia e Doenças Tropicais, 26/27:131-138.
GARCIA-ZAPATA, M. T. A. \& MARSDEN, P. D., 1986. Chagas' disease. Clinics in Tropical Medicineand Communicable Diseases, 1:557-585.

IBGE (Instituto Brasileiro de Geografia e Estatística), 1984. Atlas do Maranhão. Rio de Janeiro: IBGE.

JURBERG, J. \& GALVÃO, C., 1997. Hermanlentia n. gen. da tribo Triatomini, com um rol de espécies de Triatominae (Hemíptera, Reduviidae). Memórias do Instituto Oswaldo Cruz, 92:181-185

LENT, H. \& WYGODZINSKY, P., 1979. Revision of the Triatominae (Hemiptera, Reduviidae), and their significance as vectors of Chagas' Disease. Bulletin of the American Museum of Natural History, 163:127-520.

MARSDEN, P.; GARCIA-ZAPATA, M. T.; CASTILLO, E. A. S.; PRATA, A. R. \& MACEDO, V. O., 1994. Los primeros años del control de la enfermedad de Chagas en Mambaí, Goiás, Brasil, 1980-1992. Boletín dela Oficina Sanitaria Panamericana, 116: 111-117.

SCHOFIELD, C. J., 1985. Control of Chagas' disease. British Medical Bulletin, 41:187-194. 